\title{
Article \\ Definitive Local Therapy Is Associated with Improved Survival in Metastatic Soft Tissue Sarcomas
}

\author{
Vishruth K. Reddy ${ }^{1}$ D, Varsha Jain ${ }^{1}$, Sriram Venigalla ${ }^{1}$, Vivek Nimgaokar ${ }^{2}$, Ashwin Amurthur ${ }^{2}$, \\ Daniel Y. Lee ${ }^{2}$, Ronnie A. Sebro ${ }^{3,4,5,6}$, Robert G. Maki ${ }^{7}$, Robert J. Wilson II ${ }^{6}$, Kristy L. Weber ${ }^{6}$ and \\ Jacob E. Shabason ${ }^{1, *}$
}

\section{check for}

updates

Citation: Reddy, V.K.; Jain, V.; Venigalla, S.; Nimgaokar, V.; Amurthur, A.; Lee, D.Y.; Sebro, R.A.; Maki, R.G.; Wilson, R.J., II; Weber, K.L.; et al. Definitive Local Therapy Is Associated with Improved Survival in Metastatic Soft Tissue Sarcomas. Cancers 2021, 13, 932. https:// doi.org/10.3390/cancers13050932

Academic Editors: Torsten Kessler and Brian A. Van Tine

Received: 16 January 2021

Accepted: 18 February 2021

Published: 24 February 2021

Publisher's Note: MDPI stays neutral with regard to jurisdictional claims in published maps and institutional affiliations.

Copyright: (c) 2021 by the authors. Licensee MDPI, Basel, Switzerland. This article is an open access article distributed under the terms and conditions of the Creative Commons Attribution (CC BY) license (https:/ / creativecommons.org/licenses/by/ $4.0 /)$.
1 Department of Radiation Oncology, University of Pennsylvania, Philadelphia, PA 19104, USA; vishruth.reddy@pennmedicine.upenn.edu (V.K.R.); varsha.jain@jefferson.edu (V.J.); sriram.venigalla@pennmedicine.upenn.edu (S.V.)

2 Perelman School of Medicine, University of Pennsylvania, Philadelphia, PA 19104, USA; vivek.nimgaonkar@pennmedicine.upenn.edu (V.N.); ashwin.amurthur@pennmedicine.upenn.edu (A.A.); danielyounghoon.lee@pennmedicine.upenn.edu (D.Y.L.)

3 Department of Biostatistics, Epidemiology and Bioinformatics, University of Pennsylvania, Philadelphia, PA 19104, USA; ronnie.sebro@pennmedicine.upenn.edu

4 Department of Genetics, University of Pennsylvania, Philadelphia, PA 19104, USA

5 Department of Radiology, University of Pennsylvania, Philadelphia, PA 19104, USA

6 Department of Orthopedic Surgery, University of Pennsylvania, Philadelphia, PA 19104, USA; robert.wilson3@pennmedicine.upenn.edu (R.J.W.II); kristy.weber@pennmedicine.upenn.edu (K.L.W.)

7 Division of Hematology/Oncology, Department of Medicine, University of Pennsylvania, Philadelphia, PA 19104, USA; robert.maki@pennmedicine.upenn.edu

* Correspondence: jacob.shabason@pennmedicine.upenn.edu; Tel.: +1-(215)-662-6515; Fax: +1-(215)-349-5445

Simple Summary: Patients with metastatic soft tissue sarcomas (STS) often receive definitive local treatment with surgery and/or radiation in addition to chemotherapy to reduce morbidity associated with local tumor progression. We hypothesized that definitive local treatment is associated with improved overall survival (OS). We utilized the National Cancer Database to assess the association between definitive local treatment and OS, and factors associated with the receipt of definitive local therapy. Compared with chemotherapy alone, receipt of any definitive local therapy was associated with improved OS (median 17.9 vs. 10.1 months). The survival benefit remained on multivariate analyses and propensity-score matched analyses, with a stepwise improvement with surgery and combined modality local therapy, specifically radiotherapy (HR: $0.77 ; p<0.001$ ), surgery (HR: 0.67 ; $p<0.001$ ), and combined surgery and radiotherapy (HR: $0.42 ; p<0.001$ ). Our study suggests that chemotherapy plus definitive local treatment is associated with a significant survival benefit compared to the standard chemotherapy alone for patients with metastatic STS.

Abstract: Background: Definitive local therapy is often utilized in patients with metastatic soft tissue sarcomas (STS) to reduce morbidity associated with local tumor progression. We hypothesize that it is associated with improved overall survival (OS). Methods: Patients with newly diagnosed metastatic STS treated with chemotherapy were identified from the National Cancer Database and dichotomized into cohorts: 1. definitive local therapy (defined as either definitive dose radiotherapy, definitive surgery, or surgery with perioperative radiotherapy) or 2. conservative therapy (defined as systemic therapy with or without palliative therapy). The association between definitive local therapy and OS, and factors associated with the receipt of definitive local therapy were assessed. Results: Total of 4180 patients were identified. Compared with the conservative therapy, receipt of any definitive local therapy was associated with improved OS (median 17.9 vs. 10.1 months). The survival benefit remained on multivariate analyses and propensity-score matched analyses, with a stepwise improvement with surgery and combined modality local therapy, specifically radiotherapy (HR: $0.77 ; p<0.001$ ), surgery (HR: $0.67 ; p<0.001$ ), and combined surgery and radiotherapy (HR: 0.42 ; $p<0.001)$. Conclusions: Analysis of a large national cancer registry of patients with metastatic STS suggests that chemotherapy plus definitive local therapy is associated with a significant survival benefit compared to the standard chemotherapy alone. 
Keywords: sarcoma; radiation; surgery; local treatment; survival

\section{Introduction}

Over 13,000 patients are diagnosed with soft tissue sarcomas (STS) in the United States every year, with approximately $15 \%$ found to have metastatic disease at the time of diagnosis $[1,2]$. The mainstay of treatment for those with metastatic disease at diagnosis is chemotherapy, with local therapy often used for palliation of symptoms [3]. However, despite advances in systemic therapeutic agents for this patient population, the overall prognosis remains poor, with an estimated 5-year overall survival (OS) of 16\% [2], and a median OS for those treated with systemic therapy estimated to be between 12 and 17 months $[4,5]$. An open question remains if improved local control has the potential to impact these outcomes.

Given the discordant evidence as to the potential benefits of locally directed therapy in those with metastatic disease [6-9], latest guidelines reflect that there is no standard, optimal management for these patients [3]. However, systemic therapy alone is frequently insufficient to mitigate progression of the disease at the primary site and corresponding morbidity [6]. Numerous retrospective and prospective studies of patients with metastatic disease across various malignancies have suggested possible survival benefits of definitive local therapy to the primary site [10-18]. As such, we sought to evaluate the potential survival benefit of definitive local therapy in patients with metastatic STS. Using the National Cancer Database (NCDB), we examined the patterns of use and survival outcomes associated with definitive local therapy in patients with newly diagnosed metastatic STS who also received chemotherapy. We hypothesized that chemotherapy with definitive local therapy is associated with improved survival compared to chemotherapy alone.

\section{Results}

\subsection{Baseline Clinical Characteristics}

A total of 4180 patients met the study inclusion criteria (Figure 1). Complete patient characteristics are shown in the Table S1. Notably, the median age of the patient cohort was 56 years (range, $18-90$ years). The majority of patients were men (55\%), Non-Hispanic white $(72 \%)$, and had tumors arising from the extremity $(37 \%)$. The majority of patients were treated with conservative therapy (59\%) rather than definitive local therapy (41\%). Of those treated with definitive local therapy, 58\% were treated with surgery, $22 \%$ with radiotherapy, and $20 \%$ with combined surgery and perioperative radiation.

\subsection{Factors Associated with Receipt of Definitive Local Therapy}

On multivariable analysis, notable sociodemographic predictors of omission of definitive therapy included Medicare insurance (OR: 0.78, 95\% CI 0.64-0.95, $p<0.05$ ) or no insurance (OR: $0.66,95 \%$ CI $0.49-0.88, p<0.01$ ) (Table 1 ). Likewise, clinical predictors of omission of definitive local therapy included those with thoracic (OR: $0.55,95 \%$ CI $0.44-0.69, p<0.001$ ) or abdominal/pelvic tumors (OR: 0.49, 95\% CI 0.42-0.57, $p<0.001$ ) (Table 1). Treatment at an academic institution (OR: 1.17, 95\% CI 1.00-1.37, $p=0.05$ ) was associated with receipt of definitive local therapy. Additional factors associated with the modality of local therapy are described in the Tables S2-S4.

\subsection{Impact of Definitive Local Therapy on Overall Survival}

Compared with conservative therapy, receipt of any definitive local therapy was associated with improved OS with a median survival of 17.9 vs. 10.1 months and a 5 -year OS rate of $15.8 \%$ vs. $6.4 \%(p<0.001)$ (Figure $2 a)$. There appeared to be a stepwise improvement in survival outcomes with more aggressive local therapy with a median survival of 14.7 months, 17.0 months, and 27.2 months for radiation, surgery, and surgery plus perioperative radiation, respectively $(p<0.001)$ (Figure $2 b)$. The survival benefit of 
definitive local therapy remained on multivariate analyses with a stepwise improvement with surgery and combined modality local therapy, specifically radiation (HR: $0.77 ; 95 \% \mathrm{CI}$, $0.67-0.87 ; p<0.001$ ), surgery (HR: $0.67 ; 95 \% \mathrm{CI}, 0.61-0.73 ; p<0.001$ ), and combined surgery and radiotherapy (HR: $0.42 ; 95 \% \mathrm{CI}, 0.36-0.48 ; p<0.001$ ) (Table 2).

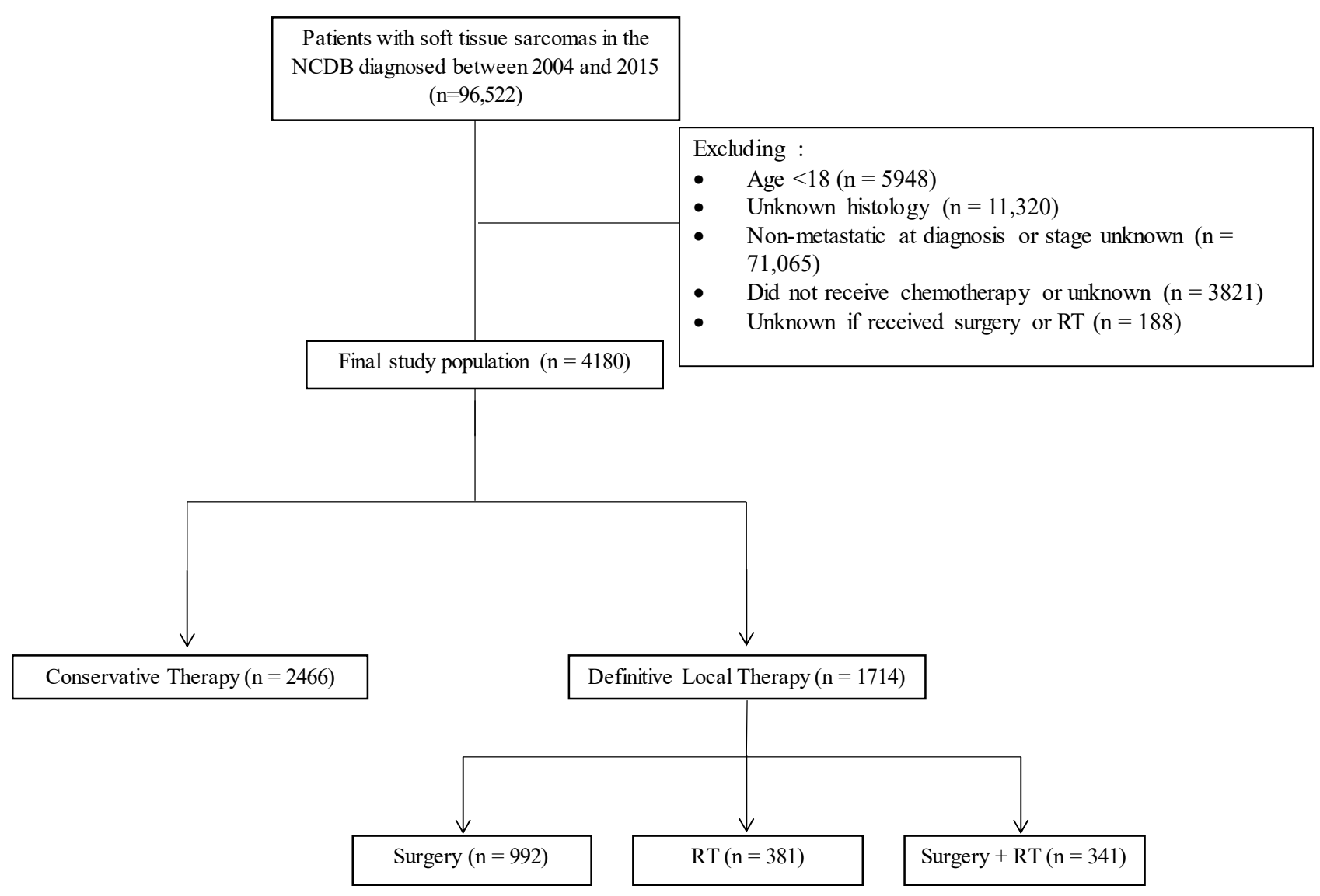

Figure 1. Consolidated standards of reporting trials (CONSORT) diagram of the patient cohort. NCDB = National Cancer Database. $\mathrm{RT}=$ radiotherapy.

Table 1. Factors associated with receipt of definitive local therapy. Univariate analysis and multivariate analysis, respectively.

\begin{tabular}{|c|c|c|c|c|}
\hline Receipt of Local Therapy & OR $(95 \%$ CI $)$ & $p$ Value & OR $(95 \%$ CI $)$ & $p$ Value \\
\hline \multicolumn{5}{|l|}{ Age } \\
\hline$<70$ years & 1 & & 1 & \\
\hline$\geq 70$ years & $0.74(0.63-0.87)$ & $<0.001$ & $0.90(0.72-1.12)$ & 0.326 \\
\hline \multicolumn{5}{|l|}{ Gender } \\
\hline Male & 1 & & - & - \\
\hline Female & $0.99(0.87-1.12)$ & 0.853 & - & - \\
\hline \multicolumn{5}{|l|}{ Race } \\
\hline Non-Hispanic White & 1 & & - & - \\
\hline Non-Hispanic Black & $0.91(0.76-1.08)$ & 0.291 & - & - \\
\hline Hispanic & $0.95(0.76-1.19)$ & 0.645 & - & - \\
\hline Other & $0.95(0.70-1.27)$ & 0.720 & - & - \\
\hline \multicolumn{5}{|l|}{ Facility Area } \\
\hline Metropolitan & 1 & & - & - \\
\hline Urban & $0.97(0.80-1.17)$ & 0.738 & - & - \\
\hline Rural & $0.74(0.45-1.22)$ & 0.240 & - & - \\
\hline Unknown & $0.92(0.66-1.28)$ & 0.632 & - & - \\
\hline
\end{tabular}


Table 1. Cont.

\begin{tabular}{|c|c|c|c|c|}
\hline Receipt of Local Therapy & OR $(95 \% \mathrm{CI})$ & $p$ Value & OR $(95 \% \mathrm{CI})$ & $p$ Value \\
\hline \multicolumn{5}{|l|}{ Facility Location } \\
\hline East & 1 & & 1 & \\
\hline South & $0.97(0.81-1.18)$ & 0.779 & $1.06(0.86-1.31)$ & 0.589 \\
\hline Central & $1.05(0.86-1.28)$ & 0.617 & $1.09(0.88-1.36)$ & 0.422 \\
\hline West & $1.23(0.98-1.55)$ & 0.077 & $1.32(1.03-1.70)$ & 0.031 \\
\hline Unknown & $1.38(1.13-1.70)$ & 0.002 & $1.36(1.04-1.78)$ & 0.027 \\
\hline \multicolumn{5}{|l|}{ Facility Type } \\
\hline Non-Academic & 1 & & 1 & \\
\hline Academic & $1.35(1.18-1.55)$ & $<0.001$ & $1.17(1.00-1.37)$ & 0.052 \\
\hline Unknown & $1.56(1.31-1.85)$ & $<0.001$ & & \\
\hline \multicolumn{5}{|l|}{ Insurance } \\
\hline Commercial & 1 & & 1 & \\
\hline Medicare & $0.71(0.62-0.83)$ & $<0.001$ & $0.78(0.64-0.95)$ & 0.012 \\
\hline Medicaid & $1.03(0.85-1.26)$ & 0.761 & $0.86(0.69-1.06)$ & 0.161 \\
\hline Uninsured & $0.75(0.57-0.98)$ & 0.035 & $0.66(0.49-0.88)$ & 0.005 \\
\hline Other & $0.83(0.57-1.21)$ & 0.342 & $0.80(0.53-1.20)$ & 0.279 \\
\hline \multicolumn{5}{|l|}{ Distance to Treatment Facility } \\
\hline$\leq 40$ miles & 1 & & 1 & \\
\hline$>40$ miles & $1.20(1.03-1.39)$ & 0.018 & $0.97(0.83-1.15)$ & 0.748 \\
\hline Unknown & $0.89(0.54-1.47)$ & 0.646 & $0.67(0.39-1.14)$ & 0.139 \\
\hline \multicolumn{5}{|l|}{ Zip Code Education Level } \\
\hline$\geq 21 \%$ & 1 & & - & - \\
\hline $13-20.9 \%$ & $1.13(0.93-1.36)$ & 0.211 & - & - \\
\hline $7-12.9 \%$ & $1.10(0.92-1.32)$ & 0.310 & - & - \\
\hline$<7 \%$ & $1.02(0.84-1.23)$ & 0.854 & - & - \\
\hline Unknown & $0.95(0.57-1.58)$ & 0.839 & - & - \\
\hline \multicolumn{5}{|l|}{ Zip Code Income Level } \\
\hline$<38,000$ & 1 & & - & - \\
\hline $38,000-47,999$ & $1.05(0.86-1.27)$ & 0.653 & - & - \\
\hline $48,000-62,999$ & $1.10(0.91-1.32)$ & 0.330 & - & - \\
\hline$\geq 63,000$ & $1.03(0.86-1.23)$ & 0.774 & - & - \\
\hline Unknown & $0.92(0.56-1.52)$ & 0.745 & - & - \\
\hline \multicolumn{5}{|l|}{ Charlson Deyo Score } \\
\hline 0 & 1 & & - & - \\
\hline 1 & $0.91(0.76-1.08)$ & 0.286 & - & - \\
\hline 2 & $0.94(0.65-1.35)$ & 0.740 & - & - \\
\hline 3 & $0.56(0.27-1.18)$ & 0.128 & - & - \\
\hline \multicolumn{5}{|l|}{ Primary Site } \\
\hline Extremity & 1 & & 1 & \\
\hline Head and Neck & $0.88(0.63-1.23)$ & 0.458 & $1.13(0.79-1.63)$ & 0.504 \\
\hline Thorax & $0.50(0.41-0.61)$ & $<0.001$ & $0.55(0.44-0.69)$ & $<0.001$ \\
\hline Abdomen/Pelvis & $0.44(0.38-0.51)$ & $<0.001$ & $0.49(0.42-0.57)$ & $<0.001$ \\
\hline Other/NOS & $0.18(0.14-0.23)$ & $<0.001$ & $0.31(0.23-0.40)$ & $<0.001$ \\
\hline \multicolumn{5}{|l|}{ Histology } \\
\hline Unclassified & 1 & & 1 & \\
\hline Undifferentiated Pleomorphic & $1.73(1.28-2.33)$ & $<0.001$ & $1.64(1.18-2.26)$ & 0.003 \\
\hline Fibrosarcoma/Myxofibrosarcoma & $2.11(1.50-2.97)$ & $<0.001$ & $2.06(1.42-2.97)$ & $<0.001$ \\
\hline Liposarcoma & $1.42(1.11-1.81)$ & 0.005 & $1.49(1.14-1.93)$ & 0.003 \\
\hline Leiomyosarcoma & $0.78(0.66-0.92)$ & 0.003 & $1.09(0.90-1.31)$ & 0.379 \\
\hline Synovial Sarcoma & $1.81(1.44-2.28)$ & $<0.001$ & $1.67(1.30-2.14)$ & $<0.001$ \\
\hline Angiosarcoma & $0.66(0.51-0.84)$ & 0.001 & $1.00(0.76-1.32)$ & 0.995 \\
\hline MPNST & $1.79(1.29-2.47)$ & $<0.001$ & $1.97(1.39-2.80)$ & $<0.001$ \\
\hline
\end{tabular}


Table 1. Cont

\begin{tabular}{|c|c|c|c|c|}
\hline Receipt of Local Therapy & OR (95\% CI) & $p$ Value & OR $(95 \% \mathrm{CI})$ & $p$ Value \\
\hline \multicolumn{5}{|l|}{ Tumor Size } \\
\hline$<5 \mathrm{~cm}$ & 1 & & 1 & \\
\hline $5.1-10 \mathrm{~cm}$ & $0.94(0.75-1.18)$ & 0.590 & $0.85(0.67-1.08)$ & 0.191 \\
\hline $10.1-15 \mathrm{~cm}$ & $1.01(0.80-1.28)$ & 0.928 & $0.87(0.68-1.12)$ & 0.280 \\
\hline$>15 \mathrm{~cm}$ & $0.96(0.76-1.21)$ & 0.727 & $0.80(0.62-1.03)$ & 0.088 \\
\hline Unknown & $0.33(0.26-0.42)$ & $<0.001$ & $0.41(0.32-0.53)$ & $<0.001$ \\
\hline \multicolumn{5}{|l|}{ Grade } \\
\hline I & 1 & & 1 & \\
\hline II & $1.17(0.69-1.99)$ & 0.566 & $1.08(0.61-1.90)$ & 0.800 \\
\hline III & $1.27(0.79-2.03)$ & 0.322 & $1.15(0.69-1.90)$ & 0.592 \\
\hline Unknown & $0.54(0.33-0.86)$ & 0.010 & $0.55(0.33-0.91)$ & 0.020 \\
\hline \multicolumn{5}{|l|}{ Year of Diagnosis } \\
\hline 2004-2007 & 1 & & 1 & \\
\hline 2008-2011 & $0.98(0.83-1.17)$ & 0.852 & $1.01(0.84-1.22)$ & 0.913 \\
\hline $2012-2015$ & $0.85(0.72-1.01)$ & 0.060 & $0.92(0.77-1.11)$ & 0.389 \\
\hline
\end{tabular}

Table 2. Survival odds in patients with metastatic STS. Univariate, multivariate, and propensity score matched analyses, respectively.

\begin{tabular}{|c|c|c|c|c|c|c|}
\hline & HR (95\% CI) & $p$ Value & HR (95\% CI) & $p$ Value & HR (95\% CI) & $p$ Value \\
\hline \multicolumn{7}{|l|}{ Local Therapy } \\
\hline None & 1 & & 1 & & 1 & \\
\hline Radiation Alone & $0.79(0.70-0.90)$ & $<0.001$ & $0.77(0.67-0.87)$ & $<0.001$ & $0.75(0.66-0.86)$ & $<0.001$ \\
\hline Surgery Alone & $0.63(0.58-0.69)$ & $<0.001$ & $0.67(0.61-0.73)$ & $<0.001$ & $0.66(0.60-0.72)$ & $<0.001$ \\
\hline Surgery and Radiation & $0.41(0.36-0.48)$ & $<0.001$ & $0.42(0.36-0.48)$ & $<0.001$ & $0.41(0.35-0.47)$ & $<0.001$ \\
\hline \multicolumn{7}{|l|}{ Age } \\
\hline$<70$ years & 1 & & 1 & & - & - \\
\hline$\geq 70$ years & $1.35(1.24-1.48)$ & $<0.001$ & $1.15(1.02-1.29)$ & 0.023 & - & - \\
\hline \multicolumn{7}{|l|}{ Gender } \\
\hline Male & 1 & & 1 & & - & - \\
\hline Female & $0.87(0.81-0.93)$ & $<0.001$ & $0.90(0.83-0.96)$ & 0.003 & - & - \\
\hline \multicolumn{7}{|l|}{ Race } \\
\hline Non-Hispanic White & 1 & & 1 & & - & - \\
\hline Non-Hispanic Black & $0.92(0.83-1.02)$ & 0.106 & $0.92(0.82-1.03)$ & 0.149 & - & - \\
\hline Hispanic & $0.68(0.59-0.79)$ & $<0.001$ & $0.73(0.63-0.86)$ & $<0.001$ & - & - \\
\hline Other & $0.88(0.73-1.04)$ & 0.138 & $0.89(0.74-1.07)$ & 0.210 & - & - \\
\hline \multicolumn{7}{|l|}{ Facility Area } \\
\hline Metropolitan & 1 & & 1 & & - & - \\
\hline Urban & $1.17(1.05-1.30)$ & 0.003 & $1.12(1.00-1.27)$ & 0.053 & - & - \\
\hline Rural & $1.14(0.86-1.51)$ & 0.351 & $1.18(0.88-1.59)$ & 0.263 & - & - \\
\hline Unknown & $1.27(1.06-1.52)$ & 0.010 & $1.05(0.83-1.31)$ & 0.704 & - & - \\
\hline \multicolumn{7}{|l|}{ Facility Location } \\
\hline East & 1 & & 1 & & - & - \\
\hline South & $0.99(0.89-1.10)$ & 0.889 & $0.92(0.82-1.04)$ & 0.179 & - & - \\
\hline Central & $1.04(0.93-1.16)$ & 0.498 & $0.99(0.88-1.11)$ & 0.852 & - & - \\
\hline West & $0.90(0.79-1.03)$ & 0.130 & $0.87(0.76-1.01)$ & 0.060 & - & - \\
\hline Unknown & $0.75(0.67-0.84)$ & $<0.001$ & $0.68(0.59-0.79)$ & $<0.001$ & - & - \\
\hline \multicolumn{7}{|l|}{ Facility Type } \\
\hline Non-Academic & 1 & & 1 & & - & - \\
\hline Academic & $0.82(0.76-0.89)$ & $<0.001$ & $0.84(0.77-0.91)$ & $<0.001$ & - & - \\
\hline Unknown & $0.68(0.62-0.75)$ & $<0.001$ & & & - & - \\
\hline
\end{tabular}


Table 2. Cont.

\begin{tabular}{|c|c|c|c|c|c|c|}
\hline & $\operatorname{HR}(95 \%$ CI) & $p$ Value & $\operatorname{HR}(95 \% \mathrm{CI})$ & $p$ Value & HR (95\% CI) & $p$ Value \\
\hline \multicolumn{7}{|l|}{ Insurance } \\
\hline Commercial & 1 & & 1 & & - & - \\
\hline Medicare & $1.34(1.24-1.46)$ & $<0.001$ & $1.13(1.01-1.25)$ & 0.026 & - & - \\
\hline Medicaid & $0.96(0.85-1.08)$ & 0.498 & $1.01(0.89-1.14)$ & 0.891 & - & - \\
\hline Uninsured & $1.03(0.88-1.20)$ & 0.725 & $1.05(0.90-1.24)$ & 0.520 & - & - \\
\hline Other & $1.06(0.86-1.32)$ & 0.563 & $1.05(0.84-1.30)$ & 0.678 & - & - \\
\hline \multicolumn{7}{|l|}{ Distance to Treatment Facility } \\
\hline$\leq 40$ miles & 1 & & 1 & & - & - \\
\hline$>40$ miles & $0.91(0.84-0.99)$ & 0.036 & $0.93(0.84-1.03)$ & 0.157 & - & - \\
\hline Unknown & $1.50(1.15-1.96)$ & 0.003 & $1.75(0.59-5.17)$ & 0.309 & - & - \\
\hline \multicolumn{7}{|l|}{ Zip Code Education Level } \\
\hline$\geq 21 \%$ & 1 & & 1 & & - & - \\
\hline $13-20.9 \%$ & $1.09(0.98-1.21)$ & 0.122 & $1.06(0.95-1.19)$ & 0.304 & - & - \\
\hline $7-12.9 \%$ & $1.09(0.98-1.21)$ & 0.097 & $1.05(0.93-1.20)$ & 0.423 & - & - \\
\hline$<7 \%$ & $1.03(0.92-1.15)$ & 0.626 & $1.02(0.88-1.19)$ & 0.757 & - & - \\
\hline Unknown & $1.55(1.18-2.04)$ & 0.002 & $0.57(0.12-2.74)$ & 0.482 & - & - \\
\hline \multicolumn{7}{|l|}{ Zip Code Income Level } \\
\hline$<38,000$ & 1 & & 1 & & - & - \\
\hline $38,000-47,999$ & $1.02(0.92-1.14)$ & 0.673 & $1.01(0.89-1.14)$ & 0.902 & - & - \\
\hline $48,000-62,999$ & $1.02(0.92-1.14)$ & 0.708 & $0.95(0.84-1.08)$ & 0.444 & - & - \\
\hline$\geq 63,000$ & $0.94(0.85-1.04)$ & 0.240 & $0.89(0.76-1.03)$ & 0.115 & - & - \\
\hline Unknown & $1.47(1.12-1.92)$ & 0.005 & $1.41(0.44-4.46)$ & 0.564 & - & - \\
\hline \multicolumn{7}{|l|}{ Charlson Deyo Score } \\
\hline 0 & 1 & & 1 & & - & - \\
\hline 1 & $1.16(1.05-1.29)$ & 0.003 & $1.08(0.97-1.20)$ & 0.151 & - & - \\
\hline 2 & $1.56(1.27-1.92)$ & $<0.001$ & $1.39(1.12-1.72)$ & 0.003 & - & - \\
\hline 3 & $2.27(1.52-3.39)$ & $<0.001$ & $2.48(1.64-3.74)$ & $<0.001$ & - & - \\
\hline \multicolumn{7}{|l|}{ Primary Site } \\
\hline Head and Neck & 1 & & 1 & & - & - \\
\hline Upper Extremity & $0.78(0.62-0.98)$ & 0.034 & $0.82(0.65-1.04)$ & 0.105 & - & - \\
\hline Lower Extremity & $0.75(0.62-0.91)$ & 0.003 & $0.78(0.64-0.95)$ & 0.014 & - & - \\
\hline Thorax & $1.12(0.91-1.38)$ & 0.281 & $1.02(0.83-1.26)$ & 0.831 & - & - \\
\hline Abdomen/Pelvis & $0.94(0.77-1.13)$ & 0.495 & $0.93(0.76-1.13)$ & 0.478 & - & - \\
\hline Other/NOS & $0.98(0.79-1.20)$ & 0.813 & $0.81(0.65-1.00)$ & 0.050 & - & - \\
\hline \multicolumn{7}{|l|}{ Histology } \\
\hline Unclassified & 1 & & 1 & & - & - \\
\hline Undifferentiated Pleomorphic & $0.91(0.77-1.07)$ & 0.247 & $0.91(0.76-1.07)$ & 0.259 & - & - \\
\hline Fibrosarcoma/Myxofibrosarcoma & $0.66(0.53-0.82)$ & $<0.001$ & $0.78(0.63-0.98)$ & 0.031 & - & - \\
\hline Liposarcoma & $0.79(0.68-0.91)$ & 0.001 & $0.86(0.74-1.00)$ & 0.050 & - & - \\
\hline Leiomyosarcoma & $0.68(0.62-0.75)$ & $<0.001$ & $0.63(0.57-0.69)$ & $<0.001$ & - & - \\
\hline Synovial Sarcoma & $0.69(0.61-0.79)$ & $<0.001$ & $0.89(0.77-1.02)$ & 0.092 & - & - \\
\hline Angiosarcoma & $1.12(0.98-1.29)$ & 0.094 & $1.06(0.92-1.22)$ & 0.440 & - & - \\
\hline MPNST & $1.00(0.84-1.20)$ & 0.976 & $1.20(1.00-1.45)$ & 0.052 & - & - \\
\hline \multicolumn{7}{|l|}{ Tumor Size } \\
\hline$<5 \mathrm{~cm}$ & 1 & & 1 & & - & - \\
\hline $5.1-10 \mathrm{~cm}$ & $1.13(0.98-1.29)$ & 0.083 & $1.18(1.03-1.36)$ & 0.017 & - & - \\
\hline $10.1-15 \mathrm{~cm}$ & $1.15(1.00-1.31)$ & 0.054 & $1.23(1.06-1.42)$ & 0.005 & - & - \\
\hline$>15 \mathrm{~cm}$ & $1.16(1.01-1.33)$ & 0.033 & $1.29(1.12-1.49)$ & 0.001 & - & - \\
\hline Unknown & $1.43(1.25-1.63)$ & $<0.001$ & $1.31(1.14-1.50)$ & $<0.001$ & - & - \\
\hline \multicolumn{7}{|l|}{ Grade } \\
\hline I & 1 & & 1 & & - & - \\
\hline II & $1.37(0.98-1.92)$ & 0.068 & $1.48(1.05-2.08)$ & 0.025 & - & - \\
\hline III & $1.93(1.43-2.61)$ & $<0.001$ & $1.98(1.45-2.69)$ & $<0.001$ & - & - \\
\hline Unknown & $1.86(1.37-2.51)$ & $<0.001$ & $1.74(1.28-2.37)$ & $<0.001$ & - & - \\
\hline
\end{tabular}


Table 2. Cont.

\begin{tabular}{ccccccc}
\hline & HR (95\% CI) & $p$ Value & HR (95\% CI) & $p$ Value & HR (95\% CI) & $p$ Value \\
\hline Year of Diagnosis & & & & & - & - \\
2004-2007 & 1 & & 1 & & - \\
$2008-2011$ & $0.90(0.83-0.99)$ & 0.026 & $0.90(0.82-0.98)$ & 0.021 & - \\
$2012-2015$ & $0.90(0.82-0.99)$ & 0.023 & $0.89(0.80-0.98)$ & 0.014 & - \\
\hline
\end{tabular}

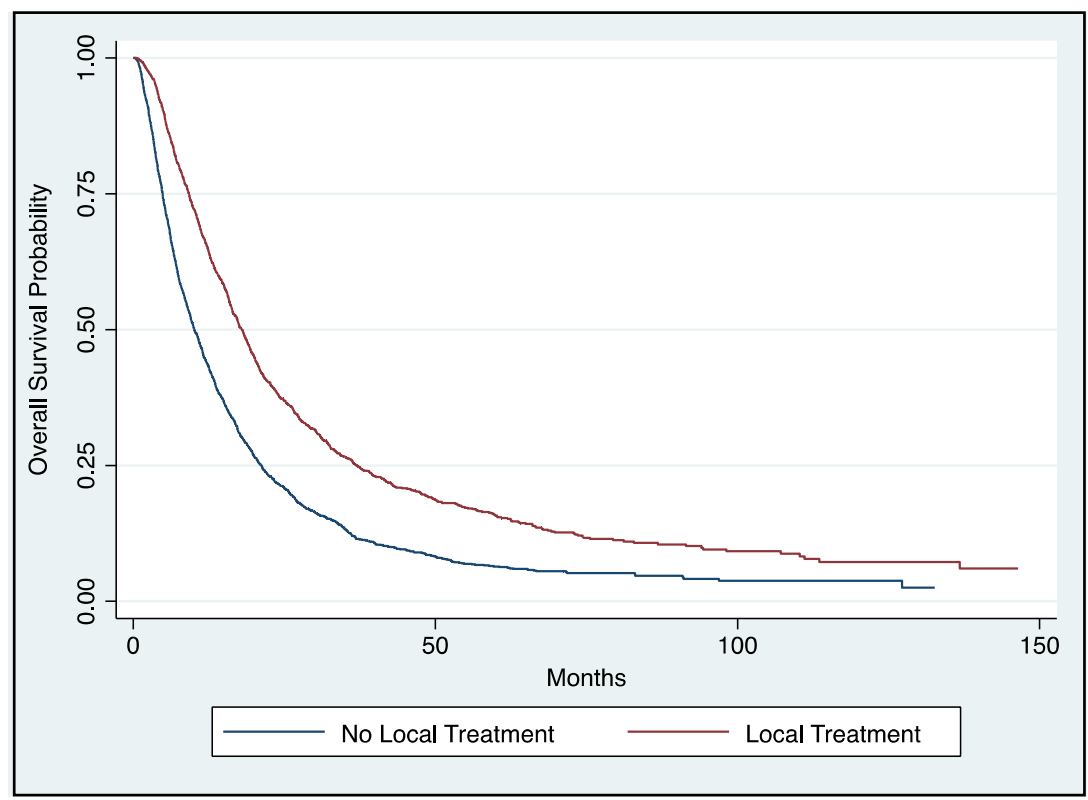

(a)

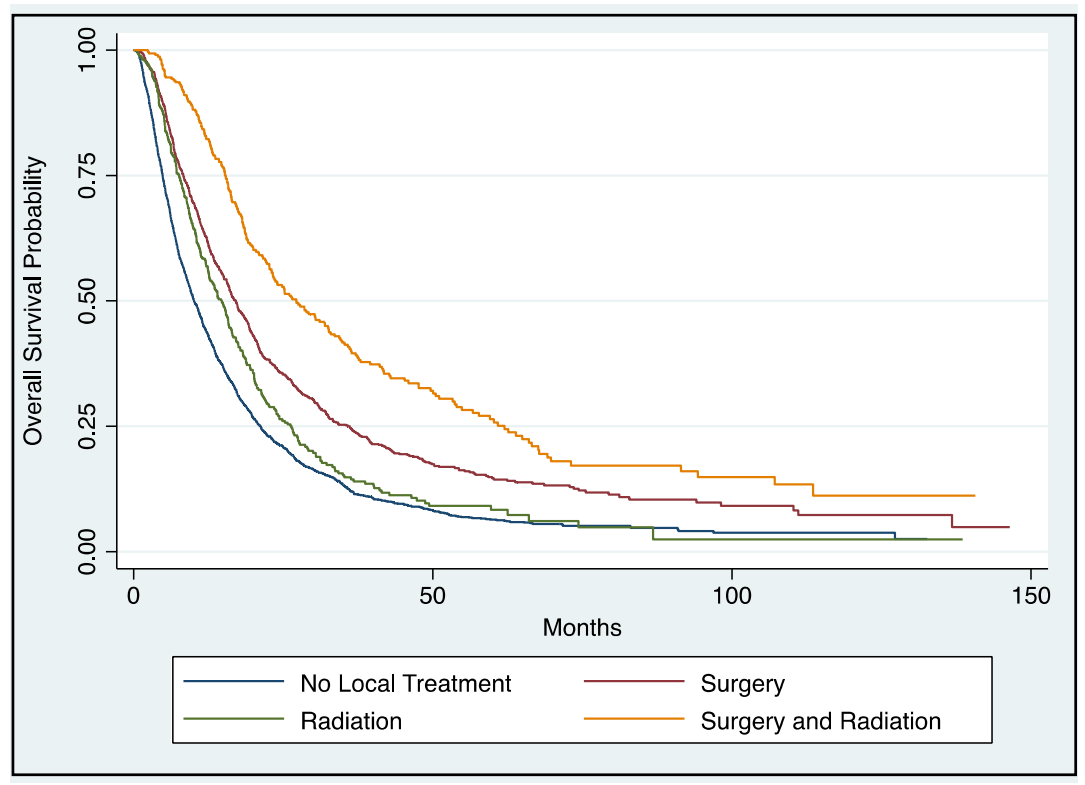

(b)

Figure 2. (a): Overall survival as a function of receipt of definitive local therapy vs. none in patients with metastatic sarcoma $(\log \operatorname{rank} p<0.001)$. (b): Overall survival as a function of receipt of definitive local therapy in patients with metastatic sarcoma $(\log \operatorname{rank} p<0.001)$.

The improvement in OS remained after PS analysis for radiation (HR: $0.75 ; 95 \%$ CI, $0.66-0.86 ; p<0.001$ ), surgery (HR: $0.66 ; 95 \%$ CI, 0.60-0.72; $p<0.001$ ), and combined surgery and radiotherapy (HR: $0.41 ; 95 \%$ CI, 0.35-0.47; $p<0.001$ ) (Table 2). Furthermore, this 
survival benefit was retained on landmark analyses at 12 months $(p<0.001)$ (Figure 3a) and 24 months $(p<0.001)$ (Figure 3b), suggesting they were unaffected by immortal time biases.

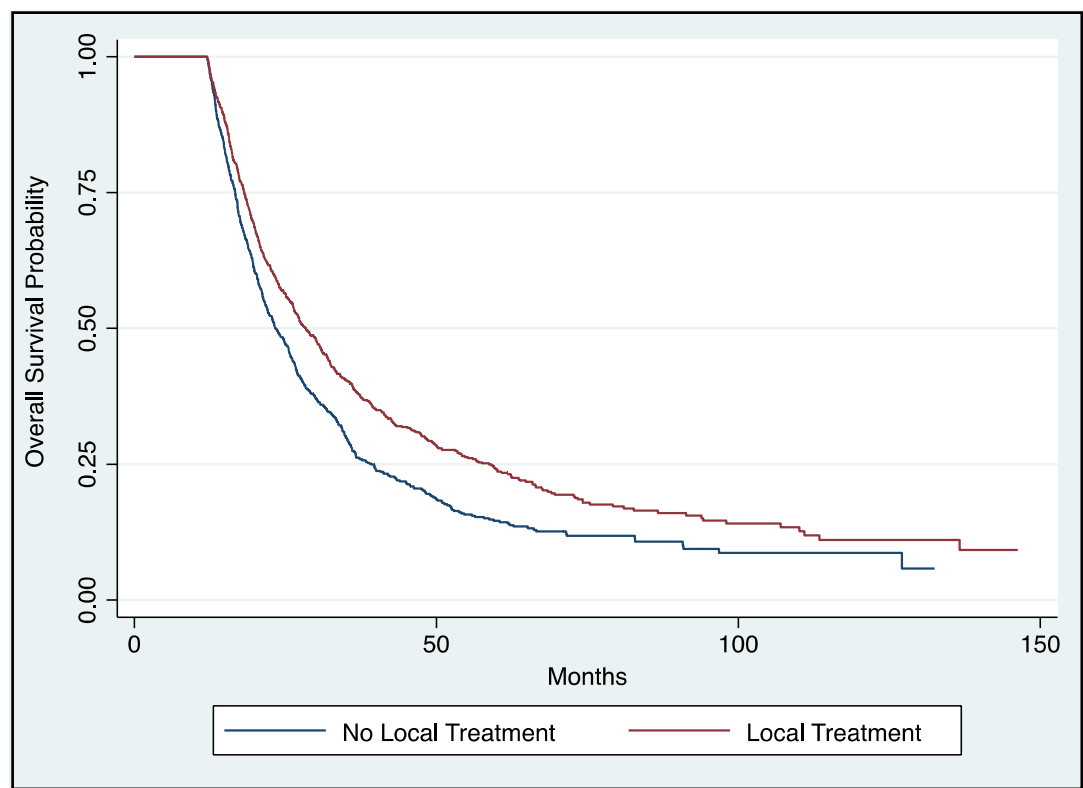

(a)

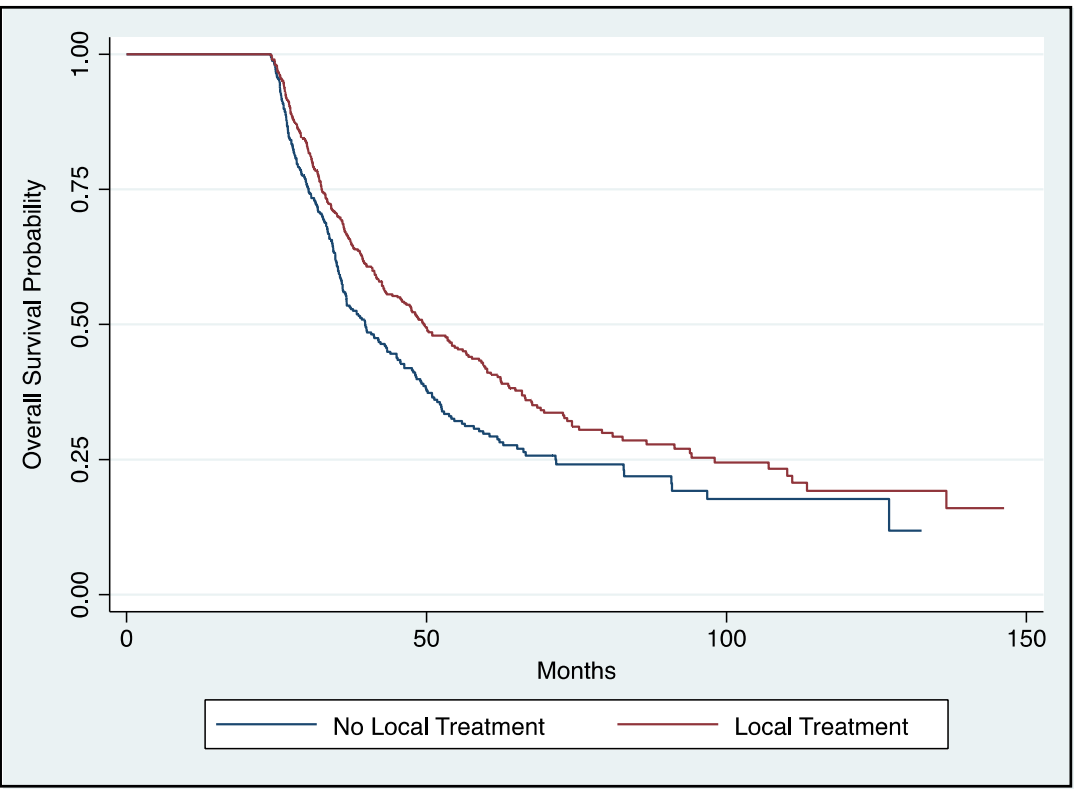

(b)

Figure 3. (a): Overall survival as a function of receipt of definitive local therapy vs. none in patients with metastatic sarcoma, those surviving $>12$ months ( $\log \operatorname{rank} p<0.001)$. (b): Overall survival as a function of receipt of definitive local therapy vs. none in patients with metastatic sarcoma, those surviving $>24$ months $(\log \operatorname{rank} p<0.001)$.

\section{Discussion}

We utilized a national cancer registry to evaluate the benefits of definitive local therapy in patients with metastatic STS. In our study we demonstrate an association between definitive local therapy and improved OS when compared with the conservative therapy alone in over 4000 patients with newly diagnosed metastatic STS. Furthermore, there appears to be an incremental survival benefit for the more aggressive local therapy. To our 
knowledge, this is the most comprehensive study to examine the benefits of definitive local therapy in this population.

National guidelines reflect that there is no standard, optimal management for patients with metastatic STS [3] given the discrepant results as to the potential benefits of locally-directed therapy [6-9]. However, the challenge remains that despite the advances in systemic therapy in recent years, these changes have translated into only incremental survival improvements, and the prognosis of this population remains poor with an estimated 5-year OS of 16\% [2,6]. Recently, there has been considerable interest in the potential benefits of definitive rather than palliative local therapy to the primary site even in the metastatic setting across a number of disease sites with both retrospective and prospective data demonstrating improvement in progression and overall survival for patients [10-18]. There have been many theories as to the mechanism of improvement in survival with the treatment of local disease, ranging from prevention of life-threatening complications from local progression to potential decreased risk of subsequent metastatic seeding through better control of the primary site of disease [19]. Mechanistic studies via animal models have suggested that the benefits of primary site cytoreduction with definitive treatment may be due to a reversal of tumor-associated immunosuppression and corresponding improvement in the host immune defenses directed at the metastatic sites of disease [20].

While we demonstrate a benefit associated with definitive local therapy for metastatic STS, it is important to highlight that such therapy is likely not the optimal treatment option for all patients with metastatic disease. Indeed, this treatment may be best employed in a carefully selected group of patients with good performance status and a low-to-moderate metastatic disease burden. Therefore, careful selection in regards to aggressive therapy is vital, particularly in patients with incurable disease, as potential for overtreatment exists and can certainly be harmful.

Strengths of the present study include a modern cohort of patients treated for STS and adjustment for a range of patient- and facility-level variables. Our study has several notable limitations given its retrospective design and reliance on the content and accuracy of information included in the NCDB. Additionally, there is inherent selection bias associated with the retrospective nature of this analysis. It is also possible that we were unable to account for the several unmeasured confounders such as patient preferences, physician attitudes, referral patterns, and quality of care received, which impacted patient selection and management. Importantly, we cannot determine the burden of metastatic disease or initial response to systemic therapy. These clinical factors certainly had a major impact on which patients were deemed appropriate for definitive local therapy. Despite these limitations, however, we aimed to more robustly account for the baseline difference between cohorts with propensity score matching, with our results demonstrating that the survival benefit associated with definitive local therapy remained. Additionally, our study suggest that this benefit was also unaffected by immortal time biases as evidenced by the results of our landmark analysis of patients who survived at least 24 months.

\section{Materials and Methods}

\subsection{Data Source}

The study population was identified from the National Cancer Database (NCDB), a national cancer registry jointly sponsored by the American College of Surgeons and the American Cancer Society that draws upon hospital registry data from more than 1500 Commission on Cancer (CoC)-accredited facilities in the United States [21,22]. The dataset captures more than $70 \%$ of incident cancers and comprises more than 34 million unique cancer cases [21,22]. Data are collected prospectively from Commission on Canceraccredited program cancer registries with nationally standardized data-coding definitions.

\subsection{Study Population}

Inclusion criteria (Figure 1) for the cohort consisted of patients $\geq 18$ years with newly diagnosed metastatic STS treated with systemic therapy, which remains the most common 
approach in the metastatic setting [3]. Metastatic disease was defined as per the American Joint Committee on Cancer staging system definition of M1 disease. Patients could have received local therapy with either radiation therapy, surgery, or surgery with perioperative radiation which reflects guideline-based suggestions for patients amenable to or requiring local therapy [3]. Patients with STS arising in the head, neck, extremities, thorax, trunk, abdomen, and pelvis and those with common adult STS histologies such as undifferentiated or unclassified histology, undifferentiated pleomorphic sarcoma, liposarcoma, leiomyosarcoma, fibrosarcoma, synovial sarcoma, angiosarcoma, and malignant peripheral nerve sheath tumor were included. Gastrointestinal stromal tumors were not included.

\subsection{Patient Cohorts and Variables}

The overall patient cohort was dichotomized into definitive local therapy and conservative therapy cohorts. Definitive local therapy was defined as the receipt of either surgery, definitive radiation to $>4400 \mathrm{cGy}$, or combined modality surgery and radiotherapy in addition to systemic therapy. Conservative therapy was defined as the receipt of systemic therapy with or without a palliative dose ( $\leq 4400 \mathrm{cGy}$ ) of radiation. The covariates examined included sex, age, race, population density of patient residence (classified as metropolitan, urban, or rural from data published by the USDA Economic Research Service), facility geographic location, facility type (nonacademic or academic), with academic referring to academic/research programs including NCI-designated comprehensive cancer centers), distance to treatment facility (calculated by distance between patient's zip code center and hospital street address), educational attainment (defined as percentage of population in patient's ZIP code without a high school degree, which is derived from the US Census data), income (defined as the median income in patient's ZIP code), Charlson/Deyo comorbidity score (a validated, weighted measure of comorbidity of patients) [23], primary site of tumor, tumor size, tumor grade, and year of treatment.

\subsection{Endpoints}

The primary endpoint was OS, which was defined as the time from the date of the initial diagnosis until death or last follow-up. We also assessed the patterns of use of definitive local therapy relative to conservative therapy.

\subsection{Statistical Analysis}

Baseline characteristics of the study cohorts were compared using Pearson's chisquared tests. All covariates achieving a threshold significance of $p<0.1$ on univariate analysis were included in the multivariable logistic regression model to assess the independent effect of each covariate on the odds of being treated with definitive local therapy relative to conservative therapy. A Cox proportional hazards regression model was created to assess the independent effect of definitive local therapy on OS compared with conservative therapy. The Kaplan-Meier estimator and log-rank test were used to compare OS between the cohorts. To more robustly account for baseline difference between cohorts, a secondary survival analysis was performed using propensity score (PS) matched cohorts for those treated with definitive local therapy. Those treated with definitive local therapy were matched to those treated with conservative therapy. This was done using 1-to-1 nearest neighbor matching without replacement [24] (matched for all covariates listed in Table 2). Absolute standardized differences of $<0.1$ between baseline covariates following matching were accepted as a measure of adequate balance [25]. A Cox survival analysis was then repeated on the matched cohorts to estimate the hazard of death associated with receipt of definitive local therapy. A landmark analysis [26] of patients who survived at least 24 months was conducted to account for immortal time bias. A two-tailed $p$-value $<0.05$ was considered statistically significant. Statistical analyses were performed using Stata SE, version 15.0 (StataCorp, College Station, TX, USA). 


\section{Conclusions}

In conclusion, our results demonstrate an association between definitive local therapy and improved OS in a cohort of patients with newly diagnosed metastatic STS. This study adds to a growing body of literature across numerous cancer sites in support of the selective use of definitive local therapy as a potentially beneficial therapeutic strategy in the setting of metastatic disease. It highlights that local control should be carefully considered, while understanding that patient comorbidities may play an equal, if not more important, role in the ultimate decision of how to manage these patients. Indeed, in our patient cohort, the majority of patients (59\%) did not receive definitive local treatment, perhaps because certain clinical factors or comorbidities rendered them suboptimal candidates and because of conflicting prior data with regards to the potential benefits of local treatment. It is important to consider that definitive local therapy can certainly come with treatment toxicity and impact patients' quality of life. Prospective validation of these findings is warranted to help establish optimal patient selection for definitive therapy and potentially improve outcomes for those with metastatic STS.

Supplementary Materials: The following are available online at https: / /www.mdpi.com/2072-6 694/13/5/932/s1, Table S1: Baseline characteristics of patient cohort, Table S2: Factors associated with receipt of surgery as definitive local treatment, Table S3: Factors associated with receipt of radiotherapy as definitive local treatment, Table S4: Factors associated with receipt of surgery and radiotherapy as definitive local treatment.

Author Contributions: Conceptualization, V.K.R. and J.E.S.; methodology, V.K.R. and J.E.S.; software, V.K.R. and J.E.S.; validation, V.K.R., V.J., S.V., and J.E.S.; formal analysis, V.K.R., V.J., S.V., and J.E.S.; investigation, V.K.R. and J.E.S.; resources, V.K.R. and J.E.S.; data curation, V.K.R. and J.E.S.; writingoriginal draft preparation, V.K.R., V.J., S.V., V.N., A.A., D.Y.L., R.A.S., R.G.M., R.J.W.II, K.L.W., J.E.S.; writing—review and editing, V.K.R., V.J., S.V., V.N., A.A., D.Y.L., R.A.S., R.G.M., R.J.W.II, K.L.W., J.E.S.; visualization, V.K.R., V.J., S.V., V.N., A.A., D.Y.L., R.A.S., R.G.M., R.J.W.II, K.L.W., J.E.S.; supervision, V.K.R. and J.E.S.; project administration, V.K.R. and J.E.S. All authors have read and agreed to the published version of the manuscript.

Funding: This research received no external funding.

Institutional Review Board Statement: Not applicable.

Informed Consent Statement: Not applicable.

Data Availability Statement: The dataset was obtained from the National Cancer Database. It is available to COC-accredited institutions upon request, as detailed at https:/ / www.facs.org/qualityprograms/cancer/ncdb/puf (accessed on 9 January 2021).

Conflicts of Interest: The authors declare no conflict of interest.

\section{References}

1. Siegel, R.L.; Miller, K.D.; Jemal, A. Cancer statistics, 2019. CA Cancer J. Clin. 2019, 69, 7-34. [CrossRef] [PubMed]

2. Surveillance, Epidemiology, and End Results Program. SEER Cancer Statistics Review. Available online: https://seer.cancer.gov/ (accessed on 15 January 2020).

3. NCCN Guidelines: Soft Tissue Sarcoma. NCCN Guidelines Version 6.2019 Soft Tissue Sarcoma. 2019. Available online: https:/ / www.nccn.org (accessed on 15 January 2020).

4. Judson, I.; Verweij, J.; Gelderblom, H.; Hartmann, J.T.; Schöffski, P.; Blay, J.Y.; Kerst, J.M.; Sufliarsky, J.; Whelan, J.; Hohenberger, P.; et al. Doxorubicin alone versus intensified doxorubicin plus ifosfamide for first-line treatment of advanced or metastatic soft-tissue sarcoma: A randomised controlled phase 3 trial. Lancet Oncol. 2014, 15, 415-423. [CrossRef]

5. Ryan, C.W.; Merimsky, O.; Agulnik, M.; Blay, J.Y.; Schuetze, S.M.; Van Tine, B.A.; Jones, R.L.; Elias, A.D.; Choy, E.; Alcindor, T.; et al. PICASSO III: A phase III, placebo-controlled study of doxorubicin with or without palifosfamide in patients with metastatic soft tissue sarcoma. J. Clin. Oncol. 2016, 34, 3898-3905. [CrossRef]

6. Kane, J.M.; Finley, J.W.; Driscoll, D.; Kraybill, W.G.; Gibbs, J.F. The treatment and outcome of patients with soft tissue sarcomas and synchronous metastases. Sarcoma 2002, 6, 69-73. [CrossRef] [PubMed]

7. Ferguson, P.C.; Deheshi, B.M.; Chung, P.; Catton, C.N.; O’Sullivan, B.; Gupta, A.; Griffin, A.M.; Wunder, J.S. Soft tissue sarcoma presenting with metastatic disease: Outcome with primary surgical resection. Cancer 2011, 117, 372-379. [CrossRef] 
8. Okiror, L.; Peleki, A.; Moffat, D.; Bille, A.; Bishay, E.; Rajesh, P.; Steyn, R.; Naidu, B.; Grimer, R.; Kalkat, M. Survival following Pulmonary Metastasectomy for Sarcoma. Thorac. Cardiovasc. Surg. 2016, 64, 146-149. [CrossRef] [PubMed]

9. Chudgar, N.P.; Brennan, M.F.; Tan, K.S.; Munhoz, R.R.; D’Angelo, S.P.; Bains, M.S.; Huang, J.; Park, B.J.; Adusumilli, P.S.; Tap, W.D.; et al. Is Repeat Pulmonary Metastasectomy Indicated for Soft Tissue Sarcoma? Ann. Thorac. Surg. 2017, 104, $1837-1845$. [CrossRef] [PubMed]

10. Guttmann, D.M.; Mitra, N.; Bekelman, J.; Metz, J.M.; Plastaras, J.; Feng, W.; Swisher-McClure, S. Improved Overall Survival with Aggressive Primary Tumor Radiotherapy for Patients with Metastatic Esophageal Cancer. J. Thorac. Oncol. 2017, 12, 1131-1142. [CrossRef] [PubMed]

11. Flanigan, R.C.; Salmon, S.E.; Blumenstein, B.A.; Bearman, S.I.; Roy, V.; McGrath, P.C.; Caton, J.R.; Munshi, N.; Crawford, E.D. Nephrectomy followed by interferon alfa-2b compared with interferon alfa-2b alone for metastatic renal-cell cancer. N. Engl. J. Med. 2001, 345, 1655-1659. [CrossRef] [PubMed]

12. Mickisch, G.H.J.; Garin, A.; Van Poppel, H.; De Prijck, L.; Sylvester, R. Radical nephrectomy plus interferon-alfa-based immunotherapy compared with interferon alfa alone in metastatic renal-cell carcinoma: A randomised trial. Lancet 2001, 358, 966-970. [CrossRef]

13. Seisen, T.; Sun, M.; Leow, J.J.; Preston, M.A.; Cole, A.P.; Gelpi-Hammerschmidt, F.; Hanna, N.; Meyer, C.P.; Kibel, A.S.; Lipsitz, S.R.; et al. Efficacy of High-Intensity Local Treatment for Metastatic Urothelial Carcinoma of the Bladder: A Propensity Score-Weighted Analysis From the National Cancer Data Base. J. Clin. Oncol. 2016, 34, 3529-3536. [CrossRef]

14. Rusthoven, C.G.; Jones, B.L.; Flaig, T.W.; Crawford, E.D.; Koshy, M.; Sher, D.J.; Mahmood, U.; Chen, R.C.; Chapin, B.F.; Kavanagh, B.D.; et al. Improved survival with prostate radiation in addition to androgen deprivation therapy for men with newly diagnosed metastatic prostate cancer. J. Clin. Oncol. 2016, 34, 2835-2842. [CrossRef]

15. Venigalla, S.; Guttmann, D.M.; Horne, Z.D.; Carmona, R.; Shabason, J.E.; Beriwal, S. Definitive local therapy is associated with improved overall survival in metastatic cervical cancer. Pract. Radiat. Oncol. 2018, 8, e377-e385. [CrossRef] [PubMed]

16. Gomez, D.R.; Blumenschein, G.R., Jr.; Lee, J.J.; Hernandez, M.; Ye, R.; Camidge, D.R.; Doebele, R.C.; Skoulidis, F.; Gaspar, L.E.; Gibbons, D.L.; et al. Local consolidative therapy versus maintenance therapy or observation for patients with oligometastatic non-small-cell lung cancer without progression after first-line systemic therapy: A multicentre, randomised, controlled, phase 2 study. Lancet Oncol. 2016, 17, 1672-1682. [CrossRef]

17. Gomez, D.R.; Tang, C.; Zhang, J.; Blumenschein Jr, G.R.; Hernandez, M.; Lee, J.J.; Ye, R.; Palma, D.A.; Louie, A.V.; Camidge, D.R.; et al. Local consolidative therapy vs. Maintenance therapy or observation for patients with oligometastatic non-small-cell lung cancer: Long-term results of a multi-institutional, phase II, randomized study. J. Clin. Oncol. 2019, 37, 1558-1565. [CrossRef]

18. Slotman, B.J.; van Tinteren, H.; Praag, J.O.; Knegjens, J.L.; El Sharouni, S.Y.; Hatton, M.; Keijser, A.; Faivre-Finn, C.; Senan, S. Use of thoracic radiotherapy for extensive stage small-cell lung cancer: A phase 3 randomised controlled trial. Lancet 2015, 385 , 36-42. [CrossRef]

19. Comen, E.; Norton, L.; Massagué, J. Clinical implications of cancer self-seeding. Nat. Rev. Clin. Oncol. 2011, 8, 369-377. [CrossRef]

20. Danna, E.A.; Sinha, P.; Gilbert, M.; Clements, V.K.; Pulaski, B.A.; Ostrand-Rosenberg, S. Surgical Removal of Primary Tumor Reverses Tumor-Induced Immunosuppression Despite the Presence of Metastatic Disease. Cancer Res. 2004, 64, $2205-2211$. [CrossRef] [PubMed]

21. American College of Surgeons American Cancer Society. National Cancer Database. National Cancer Database. 2017. Available online: https:/ / www.facs.org/quality-programs/cancer/ncdb (accessed on 15 January 2020).

22. Bilimoria, K.Y.; Stewart, A.K.; Winchester, D.P.; Ko, C.Y. The National Cancer Data Base: A powerful initiative to improve cancer care in the United States. Ann. Surg. Oncol. 2008, 15, 683-690. [CrossRef] [PubMed]

23. Deyo, R.A.; Cherkin, D.C.; Ciol, M.A. Adapting a clinical comorbidity index for use with ICD-9-CM administrative databases. J. Clin. Epidemiol. 1992, 45, 613-619. [CrossRef]

24. Austin, P.C. An introduction to propensity score methods for reducing the effects of confounding in observational studies. Multivar. Behav. Res. 2011, 46, 399-424. [CrossRef] [PubMed]

25. Austin, P.C. Balance diagnostics for comparing the distribution of baseline covariates between treatment groups in propensityScore matched samples. Stat. Med. 2009, 28, 3083-3107. [CrossRef] [PubMed]

26. Park, H.S.; Gross, C.P.; Makarov, D.V.; Yu, J.B. Immortal time bias: A frequently unrecognized threat to validity in the evaluation of postoperative radiotherapy. Int. J. Radiat. Oncol. Biol. Phys. 2012, 83, 1365-1373. [CrossRef] [PubMed] 